\title{
Review of: "Null results for the steal-framing effect on out-group aggression"
}

\author{
Christine Clavien ${ }^{1}$ \\ 1 University of Geneva
}

Potential competing interests: The author(s) declared that no potential competing interests exist.

This study is interesting and adds one more little stone to the explanation of human behaviour. I find it important to also publish null results. However, for the reason described below, I am not convinced that the design of the game used in this study (slight variations within the IPD-MD game) addresses the most relevant incentive structure underlying human tendencies to out-group aggression.

It seems to me that the relevant cue from most theoretical evolutionary models on group conflict/competition, is the "zero sum game" condition. The crucial incentive feature is not so much "winning more" (more for oneself or more than the other group - which is conductive to selfish psychological mechanisms), but "competing for limited resources" (which is more conductive to reactive safeguard psychological mechanisms). This also makes sense in light of human evolutionary past. If a group enters in a natural dynamic of growth (more offspring than deaths within the group) or if an external event reduces the available resources (e.g. a cold year leading to less available preys or bad vegetation growth), to secure in-group's well-being or survival, it becomes crucial to access new resources or to avoid resources being taken by other groups. These situations must have been frequent in the evolutionary past. If on the contrary resources are available (e.g. a good year, discovery of new resources) the zero sum game condition is alleviated and the structure of the game changes: in such situations, there is no reasons to "want to" keep more for oneself (even if de facto we happen to have more for ourselves). These circumstances must also have happened regularly. Thus it may not be evolutionary advantageous to acquire broad-scope selfish psychological tendencies or broad-scope tendencies to be aggressive or overcompetitive towards outgroup members because our ancestors evolved in a complex world and had to adjust their behaviour to different game structures. I would rather be interested in laboratory experiments manipulating the zero sum condition: thus exploring the tendency to become increasingly selfish and competitive towards outgroup individuals depending on the increase of resource scarcity.

More generally, I also would like to highlight how tentative and risky it is to infer (or reject) the presence of a psychological tendency from results of one model (or group of similar models). Showing the stability of a behavioural strategy in a model does not yet imply that humans have evolved symmetric psychological tendencies. Further evidences are needed to strengthen that new hypothesis. Notably we need to show that (1) our ancestors were repetitively kept in decision-making situations corresponding the decisionmaking structure imposed by the model, (2) had no available way out, and (3) that the adaptive behaviour 
in that decision-making structure had no significant negative side-effects in other relevant domains of life (e.g. stealing leads to direct gain but may negatively impact people's reputation on the long run).

Methodologically, as recognized by the authors, I am worried about the sample sizes used in the study. It is increasingly acknowledged that studies with small sample sizes are conductive to both false positive and false negative results. Moreover, within such experimental setting, it is difficult to control for the quality of the data (i.e. exclude participants that did not do the task seriously). The authors report having conducted an understanding check, but they did not use this check for excluding the $10 \%$ participants who failed to respond correctly. 